\title{
One view or two views for wide-angle tomosynthesis with synthetic mammography in the assessment setting?
}

\author{
Paola Clauser $^{1}$ (D) Pascal A. T. Baltzer ${ }^{1} \cdot$ Panagiotis Kapetas $^{1} \cdot$ Ramona Woitek $^{1} \cdot$ Michael Weber $^{2} \cdot$ Federica Leone $^{3}$. \\ Maria Bernathova ${ }^{1} \cdot$ Thomas H. Helbich $^{1}$
}

Received: 22 January 2021 / Revised: 14 April 2021 / Accepted: 19 May 2021 / Published online: 29 July 2021

(C) The Author(s) 2021

\begin{abstract}
Objectives To evaluate the diagnostic performance in the assessment setting of three protocols: one-view wide-angle digital breast tomosynthesis (WA-DBT) with synthetic mammography (SM), two-view WA-DBT/SM, and two-view digital mammography (DM).

Methods Included in this retrospective study were patients who underwent bilateral two-view DM and WA-DBT. SM were reconstructed from the WA-DBT data. The standard of reference was histology and/or 2 years follow-up. Included were 205 women with 179 lesions ( 89 malignant, 90 benign). Four blinded readers randomly evaluated images to assess density, lesion type, and level of suspicion according to BI-RADS. Three protocols were evaluated: two-view DM, one-view (mediolateral oblique) WA-DBT/SM, and two-view WA-DBT/SM. Detection rate, sensitivity, specificity, and accuracy were calculated and compared using multivariate analysis. Reading time was assessed.

Results The detection rate was higher with two-view WA-DBT/SM $(p=0.063)$. Sensitivity was higher for two-view WA-DBT/ SM compared to two-view DM $(p=0.001)$ and one-view WA-DBT/SM $(p=0.058)$. No significant differences in specificity were found. Accuracy was higher with both one-view WA-DBT/SM and two-view WA-DBT/SM compared to DM $(p=0.003$ and $>0.001$, respectively). Accuracy did not differ between one- and two-view WA-DBT/SM. Two-view WA-DBT/SM performed better for masses and asymmetries. Reading times were significantly longer when WA-DBT was evaluated.

Conclusions One-view and two-view WA-DBT/SM can achieve a higher diagnostic performance compared to two-view DM. The detection rate and sensitivity were highest with two-view WA-DBT/SM. Two-view WA-DBT/SM appears to be the most appropriate tool for the assessment of breast lesions.

Key Points

- Detection rate with two-view wide-angle digital breast tomosynthesis (WA-DBT) is significantly higher than with two-view digital mammography in the assessment setting.

- Diagnostic accuracy of one-view and two-view WA-DBT with synthetic mammography (SM) in the assessment setting is higher than that of two-view digital mammography.

- Compared to one-view WA-DBT with SM, two-view WA-DBT with SM seems to be the most appropriate tool for the assessment of breast lesions.
\end{abstract}

Keywords Breast cancer $\cdot$ Mammography $\cdot$ Digital breast tomosynthesis $\cdot 3 \mathrm{D}$ mammography

Paola Clauser

clauser.p@hotmail.it

1 Department of Biomedical Imaging and Image-Guided Therapy, Medical University of Vienna/General Hospital Vienna, Waehringer Guertel 18-20, Vienna, Austria
2 Division of General and Pediatric Radiology, Department of Biomedical Imaging and Image-Guided Therapy, Medical University of Vienna, Waehringer Guertel 18-20, 1090 Vienna, Austria

3 Ospedale Luigi Sacco - Polo Universitario, via G.B. Grassi 74, 20157 Milan, Italy 


\section{Abbreviations \\ ACR American College of Radiology \\ BI-RADS Breast Imaging Reporting and Data System \\ DM Digital mammography \\ GEE Generalized estimating equations \\ SM Synthetic mammography \\ WA-DBT Wide-angle digital breast tomosynthesis}

\section{Introduction}

Digital breast tomosynthesis (DBT) is emerging as the standard imaging modality for breast cancer diagnosis, based on improvements in both diagnostic and screening imaging outcomes [1-7]. Concerns over increased radiation dose [8,9] have prompted the development of synthetic mammography (SM) in which two-dimensional images are reconstructed from the DBT data to replace the full-field digital mammography (DM) portion of the examination [10].

Image acquisition for DBT occurs with the x-ray tube moving in an arc, which varies, depending on the manufacturer, between $15^{\circ}$ (narrow angle) and $50^{\circ}$ (wide angle) [11]. In general, a wide-angle acquisition results in more tomographic information and yields a better vertical ( $\mathrm{z}$ axis) resolution and delineation of soft tissues [12]. Based on the improved resolution of wide-angle systems, several studies have been performed that suggest the use of one-view (mediolateral oblique) wide-angle DBT (WA-DBT) only, with or without the second-view (cranio-caudal) DM [13-15]. The rationale is that the additional quasi-3D information of a one-view WADBT might obviate the need for a second view, which is currently also considered essential when using DBT. The one-view WA-DBT strategy would allow a further reduction of the radiation dose and add to the reduction already achieved with SM. In addition, this could lead to a reduction in reading time, as well as a reduction in radiologists' fatigue when reading two-view DBT [16-18].

To test this hypothesis, we performed a retrospective study to evaluate the detection rate and diagnostic performance of one-view (mediolateral oblique) WA-DBT combined with SM, compared to a two-view WA-DBT combined with SM, and two-view DM alone in an assessment setting.

\section{Materials and methods}

This retrospective study was approved by the ethics committee of our university. The need for written informed consent for the use of routine medical data records was waived. The study was performed in accordance with the Declaration of Helsinki statement for medical research involving human subjects.
Eligible subjects were patients who underwent two-view DM and WA-DBT because of inconclusive or suspicious findings seen during a screening examination on mammography and/or ultrasound (based on the Breast Imaging Reporting and Data System [BI-RADS] 0, 4, or 5 category) between March and June 2015. Women who presented with symptoms (palpable lumps, nipple discharge) and women who had follow-up exams after breast cancer treated with breast-conserving surgery were also included. The exclusion criterion to generate our study cohort was the absence of a standard of reference (image-guided biopsy, surgery, or at least 24 months followup in case of negative examinations (BI-RADS 1)). Further exclusion criteria were pregnant or lactating women, women undergoing neoadjuvant chemotherapy for a known breast cancer, and women who underwent mastectomy for a previous breast cancer.

This manuscript presents additional results from a dataset of patients, which has been published previously [19].

Included in the analysis were 205 women (mean age, 56.2 years; range, 36-84): 49 had no mammographic findings (BI-RADS 1), 135 had one lesion (BI-RADS 2-5), 19 women had two lesions, and two women had three lesions. The BI-RADS 1 cases were used as confounders to perform the detection task and were not considered in the further data analysis.

\section{Image acquisition}

DM combined with WA-DBT was acquired with a commercially available system (Mammomat Inspiration, Siemens Healthineers), using the two standard views (cranio-caudal and mediolateral oblique) during the same breast compression. This device is characterized by a tungsten/rhodium anode/filter combination. The DBT view was acquired by 25 projections over an angular range of $50^{\circ}$. The DBT projections were reconstructed using EMPIRE technology (Siemens Healthineers), including statistical artifact reduction to mitigate out-of-plane artifacts and iterative filtering in image space to suppress noise. The resulting tomosynthesis slices have an in-plane resolution of $0.085 \mathrm{~mm} \times 0.085 \mathrm{~mm}$ and are $1 \mathrm{~mm}$ apart. The DBT volumes SM (Insight 2D, Siemens Healthineers) were reconstructed based on a 3D volume ray-casting method in order to obtain the exact same distribution of calcifications and the same tissue structures as in the DMs.

The average acquisition time using this system is $1 \mathrm{~s}$ for one-view DM and below $25 \mathrm{~s}$ for one-view WA-DBT. Considering also the time for positioning and reconstruction, the estimated examination time for two-view DM, one-view WA-DBT with SM, and two-view WA-DBT with SM is below $4 \mathrm{~min}, 4 \mathrm{~min}$, and $8 \mathrm{~min}$ respectively. 


\section{Image analysis}

Four radiologists with at least 7 years of experience in breast imaging performed the readings on a dedicated workstation (syngo.Breast Care, Siemens Healthineers) with highresolution monitors. Prior to data collection, all readers analyzed a series of 20 test cases with SM in order to become familiar with typical image appearance. These reconstructed, synthesized 2D images were provided by Siemens Healthcare and were not part of the study.

Each reading session included all the 205 cases presented in a randomized order with different reading protocols. Each case was displayed only once per reading session. For this study, we considered three different reading protocols: twoview WA-DBT with SM, one-view (mediolateral oblique) WA-DBT with SM, and two-view DM. The mediolateral oblique view was chosen over the cranio-caudal view as it allows the evaluation of a larger area of breast parenchyma. The reading sessions were separated by a wash-out period of at least 3 weeks to avoid memory bias. Readers were aware of the inclusion criteria of the study, but they were blinded to the clinical history of the patients, previous and additional imaging (previous mammography, US or MRI), and histology.

During each reading session, readers were asked to define breast density using the classification suggested by BI-RADS [20], define the presence or absence of one or more lesions per breast (detection task), define lesion conspicuity using a scale from 1 (not conspicuous) to 10 (very conspicuous), define lesion type (mass, calcifications, architectural distortion, and asymmetry), and assign a BI-RADS category [20] to each detected lesion. Reading times were measured from the timepoint the case was available for review to the timepoint a BI-RADS category was assigned. Data on radiation exposure (average glandular dose, AGD) were collected. Lesion size was measured by a fifth reader, aware of lesion location and histology.

\section{Statistical analysis}

All statistical computations were performed using IBM SPSS Statistics for Windows version 24.0.2. Detection rate, sensitivity, specificity, and accuracy were calculated for each reader. The detection rate was measured as the number of lesions detected of the total number of lesions included, both benign and malignant. Only the cases in which a lesion was present were included in the diagnostic performance analysis. Sensitivity was measured as the number of lesions assigned a BI-RADS 4 or 5 of the total number of malignant lesions. Undetected lesions were treated as false negatives. Specificity was measured as the number of lesions assigned a BI-RADS $\leq 3$ on benign lesions detected by the reader. To compare the three protocols and the four readers in terms of lesion conspicuity, lesion detection,
Table 1 Histology and lesion type of the lesions included in the analysis

\begin{tabular}{ll}
\hline Histology & Number of lesions (\%) \\
\hline Malignant & $89(49.7)$ \\
Invasive carcinoma NST & $43(48.3)$ \\
Invasive carcinoma NST with DCIS & $29(32.6)$ \\
Invasive lobular carcinoma & $3(3.4)$ \\
Invasive lobular carcinoma with DCIS & $2(2.2)$ \\
Ductal carcinoma in situ & $11(12.4)$ \\
Angiosarcoma & $1(1.1)$ \\
Benign & $90(50.3)$ \\
Fibrocystic changes & $38(42.2)$ \\
Fibroadenoma & $21(23.4)$ \\
Papilloma & $17(18.9)$ \\
Adenosis & $4(4.4)$ \\
Other & $10(11.1)$ \\
Lesion type & $179(100)$ \\
Mass & $87(48.6)$ \\
Microcalcifications & $65(36.3)$ \\
Architectural distortion & $19(10.6)$ \\
Asymmetry & $8(4.5)$
\end{tabular}

NST non-special type, DCIS ductal carcinoma in situ, Other periductal mastitis, pseudoangiomatous stromal hyperplasia, hamartoma, inflammation

and diagnostic performance, generalized estimating equations (GEE) were used to obtain multiple measurements per case. A further multivariate analysis using GEE was performed to evaluate the effect of breast density and lesion type on the detection rate and diagnostic accuracy. To evaluate the effect of lesion size on the detection rate, lesions were divided into three groups (below $10 \mathrm{~mm} ; 11-20 \mathrm{~mm}$; above $21 \mathrm{~mm}$ ) and the chi-square test for trends was used to analyze the effect of lesion size on detection rate. Fleiss' kappa was used to assess inter-reader agreement for the BI-RADS category. Mixedmodel analyses of variance were used to analyze reading

Table 2 Lesion detection rate for each reader for the three reading protocols: two-view digital mammography $(2 \mathrm{v}-\mathrm{DM})$, one-view wide-angle digital breast tomosynthesis with synthetic mammography (1v-WA$\mathrm{DBT} / \mathrm{SM})$, and two-view WA-DBT with SM (2v-WA-DBT/SM)

\begin{tabular}{rlll}
\hline & $\begin{array}{l}2 \mathrm{v}-\mathrm{DM} \\
\mathrm{N}^{\circ}(\%)\end{array}$ & $\begin{array}{l}1 \mathrm{v}-\mathrm{WA}-\mathrm{DBT} / \mathrm{SM} \\
\mathrm{N}^{\circ}(\%)\end{array}$ & $\begin{array}{l}\text { 2v-WA-DBT/SM } \\
\mathrm{N}^{\circ}(\%)\end{array}$ \\
\hline Detection & & & \\
Reader 1 & $138(77.1)$ & $135(75.4)$ & $139(77.7)$ \\
Reader 2 & $123(68.7)$ & $120(67.0)$ & $131(73.2)^{*}$ \\
Reader 3 & $136(76.0)$ & $138(77.1)$ & $140(78.2)$ \\
Reader 4 & $143(79.9)$ & $144(80.4)$ & $152(84.9)^{*}$ \\
\hline
\end{tabular}

$N^{\circ}$ number of lesions detected of the total of 179 lesions included in the analysis

*Significant difference compared to $2 \mathrm{v}-\mathrm{DM}$ 
Table 3 Distribution of the ACR BI-RADS categories in the detected lesions per reader and reading protocols (two-view digital mammography $(2 \mathrm{v}-\mathrm{DM})$, one-view wide-angle digital breast tomosynthesis with synthetic mammography (1v-WA-DBT/SM), and two-view WA-DBT with SM (2v-WA-DBT/SM))

\begin{tabular}{|c|c|c|c|c|c|}
\hline Reading protocol & Reader & BI-RADS 2 & BI-RADS 3 & BI-RADS 4 & BI-RADS 5 \\
\hline \multirow[t]{4}{*}{$2 \mathrm{v}-\mathrm{DM}$} & 1 & 40 & 9 & 53 & 36 \\
\hline & 2 & 28 & 9 & 44 & 42 \\
\hline & 3 & 32 & 21 & 50 & 33 \\
\hline & 4 & 48 & 17 & 44 & 34 \\
\hline \multirow[t]{4}{*}{ 1v-WA-DBT/SM } & 1 & 40 & 17 & 40 & 38 \\
\hline & 2 & 24 & 7 & 24 & 65 \\
\hline & 3 & 25 & 28 & 37 & 48 \\
\hline & 4 & 37 & 20 & 43 & 44 \\
\hline \multirow[t]{4}{*}{ 2v-WA-DBT/SM } & 1 & 43 & 11 & 43 & 42 \\
\hline & 2 & 27 & 7 & 27 & 70 \\
\hline & 3 & 31 & 22 & 34 & 53 \\
\hline & 4 & 41 & 14 & 46 & 51 \\
\hline
\end{tabular}

times. A $p$ value $<0.05$ was considered statistically significant. For post hoc tests, Bonferroni corrections were used.

\section{Results}

Included in the analysis were 205 women with 179 lesions (89 malignant and 90 benign). Details about lesion histology and types of lesion are summarized in Table 1. Mean lesion size measured on WA-DBT was $23 \mathrm{~mm}$ (standard deviation 15.6).

\section{Detection rate}

Results are summarized in Table 2. Overall, two-view WADBT with SM achieved the highest detection rate $(78.5 \%)$, followed by two-view DM (75.4\%), and one-view WADBT with SM (75\%). GEE showed that the detection rate was dependent on the reader $(p<0.001)$. Despite this, the detection rate was higher for two-view WA-DBT with SM for all four readers. These findings were confirmed at multivariate analysis also taking lesion type into account. The detection rate was not dependent on lesion type, though the

Table 4 Diagnostic performance for each reader for the three reading protocols: two-view digital mammography (2v-DM), one-view wide-angle digital breast tomosynthesis with synthetic mammography (1v-WA-DBT/SM), and two-view WA-DBT with SM (2v-WA-DBT/SM)

\begin{tabular}{|c|c|c|c|}
\hline & $\begin{array}{l}2 \mathrm{v}-\mathrm{DM} \\
\%\left(\mathrm{~N}^{\circ}\right)\end{array}$ & $\begin{array}{l}\text { 1v-WA-DBT/SM } \\
\%\left(\mathrm{~N}^{\circ}\right)\end{array}$ & $\begin{array}{l}2 \mathrm{v}-\mathrm{WA}-\mathrm{DBT} / \mathrm{SM} \\
\%\left(\mathrm{~N}^{\circ}\right)\end{array}$ \\
\hline \multicolumn{4}{|l|}{ Sensitivity } \\
\hline Reader 1 & $77.5(69 / 89)$ & $76.4(68 / 89)$ & $79.8(71 / 89)$ \\
\hline Reader 2 & $71.9(64 / 89)$ & $82.0(73 / 89)$ & $85.4 *(76 / 89)$ \\
\hline Reader 3 & $73.0(65 / 89)$ & $80.9(72 / 89)$ & $82.0(73 / 89)$ \\
\hline Reader 4 & $67.4(60 / 89)$ & $79.8(71 / 89)$ & $85.4 *(76 / 89)$ \\
\hline \multicolumn{4}{|l|}{ Specificity } \\
\hline Reader 1 & $63.5(40 / 63)$ & $78.3(47 / 60)$ & $74.2(46 / 62)$ \\
\hline Reader 2 & $46.3(25 / 54)$ & $60.0(27 / 45)$ & $53.7(29 / 54)$ \\
\hline Reader 3 & $63.5(40 / 63)$ & $64.9(37 / 57)$ & $72.6(45 / 62)$ \\
\hline Reader 4 & $65.6(42 / 64)$ & $72.3(47 / 65)$ & $68.6(48 / 70)$ \\
\hline \multicolumn{4}{|l|}{ Accuracy } \\
\hline Reader 1 & $71.7(109 / 152)$ & $77.2(115 / 149)$ & $77.5(117 / 151)$ \\
\hline Reader 2 & $62.2(89 / 143)$ & $74.6^{*}(100 / 134)$ & $73.4 *(105 / 143)$ \\
\hline Reader 3 & $69.1(105 / 152)$ & $74.7(109 / 146)$ & $78.1(118 / 151)$ \\
\hline Reader 4 & $66.7(102 / 153)$ & $76.6(118 / 154)$ & $78.0 *(124 / 159)$ \\
\hline
\end{tabular}

*Significant difference compared to $2 \mathrm{v}$-DM 

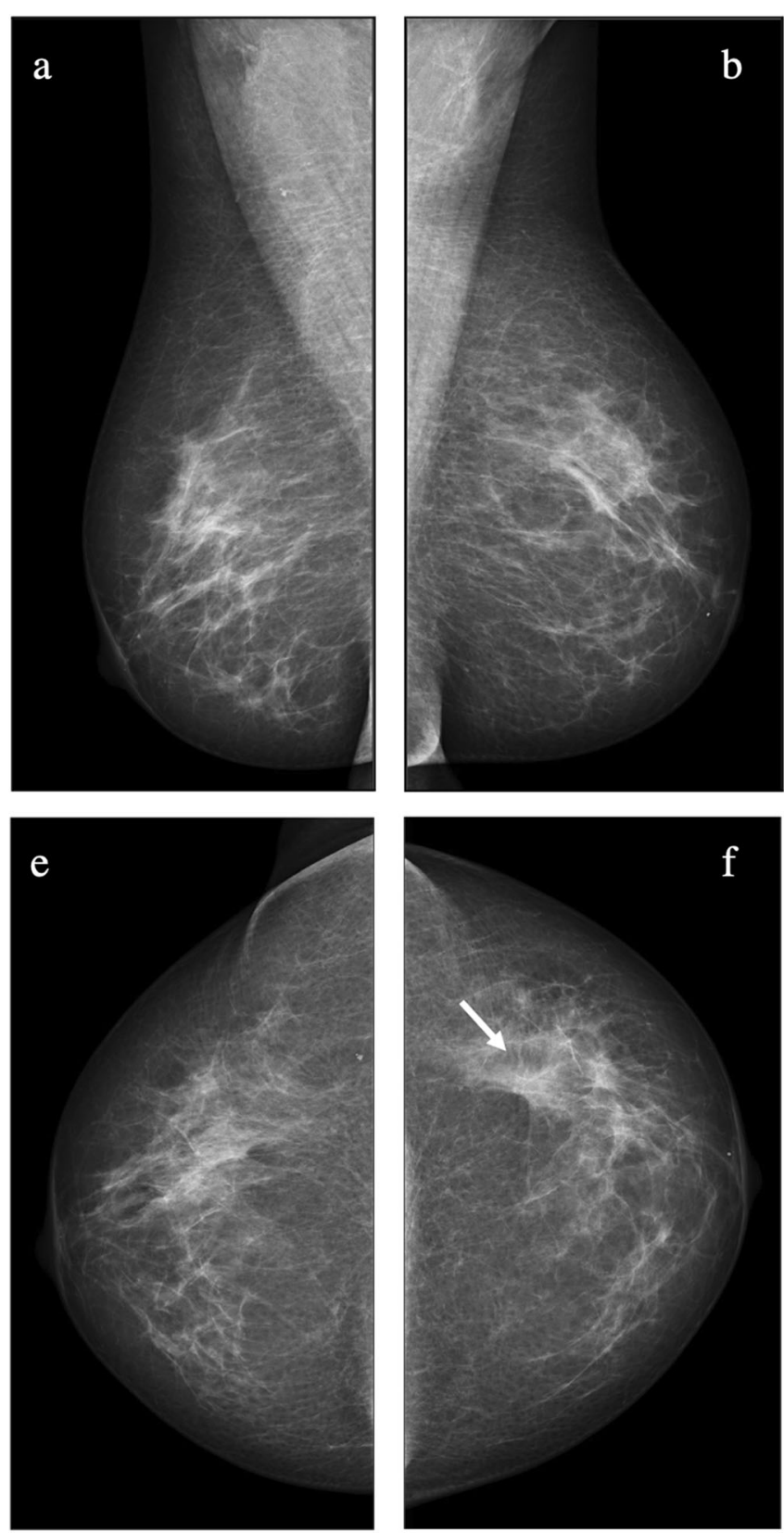

Fig. 1 A 56-year-old woman with suspicious calcifications on the right side (benign at biopsy). An architectural distortion was visible in the upper outer quadrant of the left breast (arrow). The lesion was detected and correctly classified as suspicious by one in four readers with digital mammography (DM) and with one-view wide-angle digital breast tomosynthesis with synthetic mammography (WA-DBT with SM), while

detection rate for soft-tissue findings such as architectural distortions tended to be higher for two-view WA-DBT with SM $(p=0.073)$.

The detection rate improved with increasing lesion size for all four readers when evaluating both DM $(p \leq 0.020)$ and with one-view WA-DBT with SM $(p \leq 0.041)$. The detection rate improved with increasing lesion size for only two of the four readers when evaluating two-view WA-DBT with SM $(p$ $\leq 0.008$ and $p \geq 0.404$, respectively).
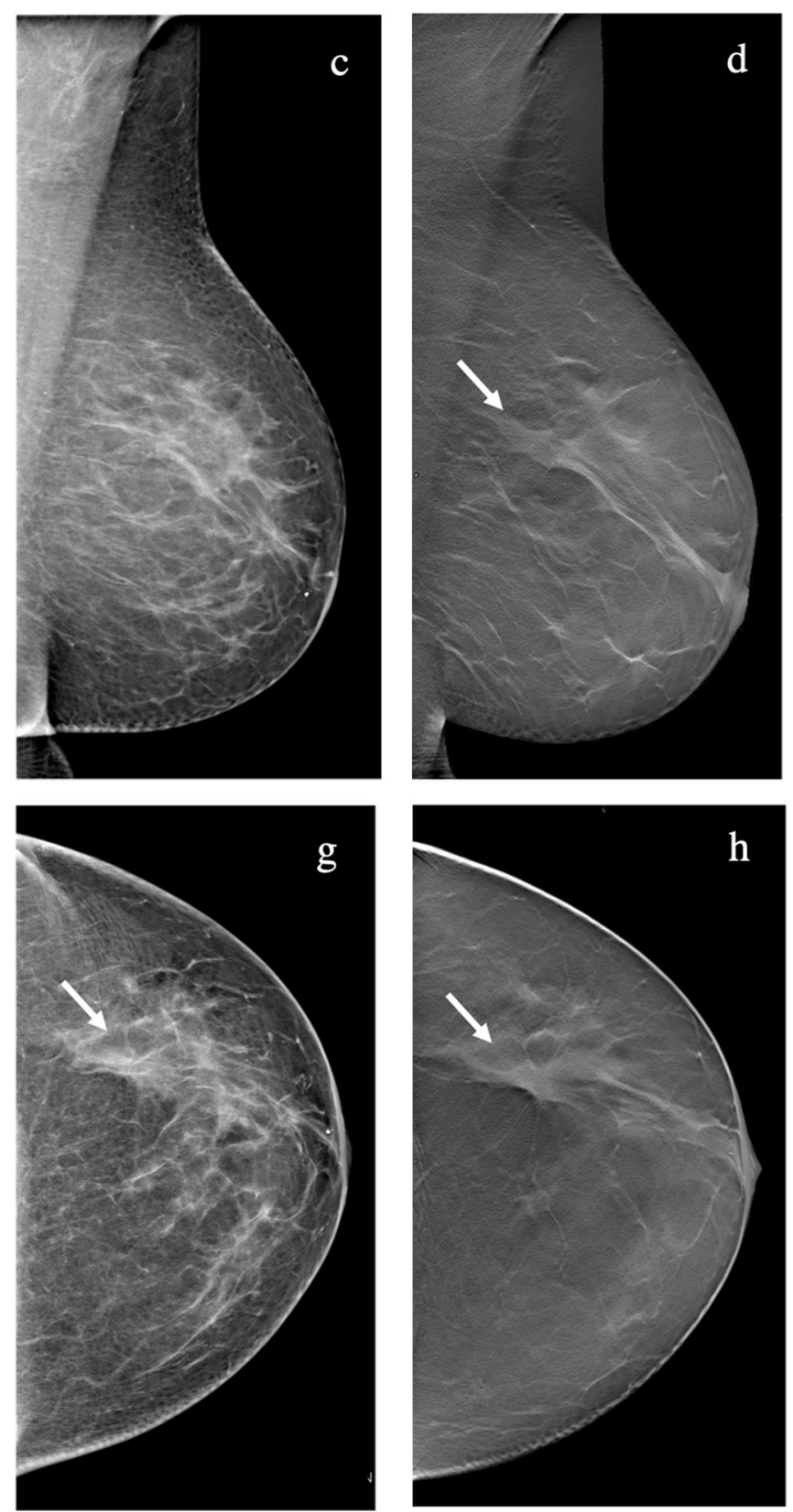

it was detected and classified as suspicious by three in four readers with two-view WA-DBT with SM. The lesion was biopsied under ultrasound guidance, and the diagnosis was invasive ductal carcinoma grade 2 with ductal carcinoma in situ (a, b DM bilateral mediolateral oblique view (MLO); c SM MLO; d DBT MLO; e, f DM bilateral cranio-caudal view (CC); g SM CC; h DBT CC)

\section{Diagnostic performance}

The BI-RADS categories assigned to the detected lesions by each reader with each reading protocol are summarized in Table 3.

Results are summarized in Table 4 . The overall sensitivity of two-view WA-DBT with SM was $83.1 \%$, and it was higher than the sensitivity of one-view WA-DBT with SM $(79.8 \%, p$ $=0.058)$ and two-view $\operatorname{DM}(72.5 \%, p=0.001)$. The 

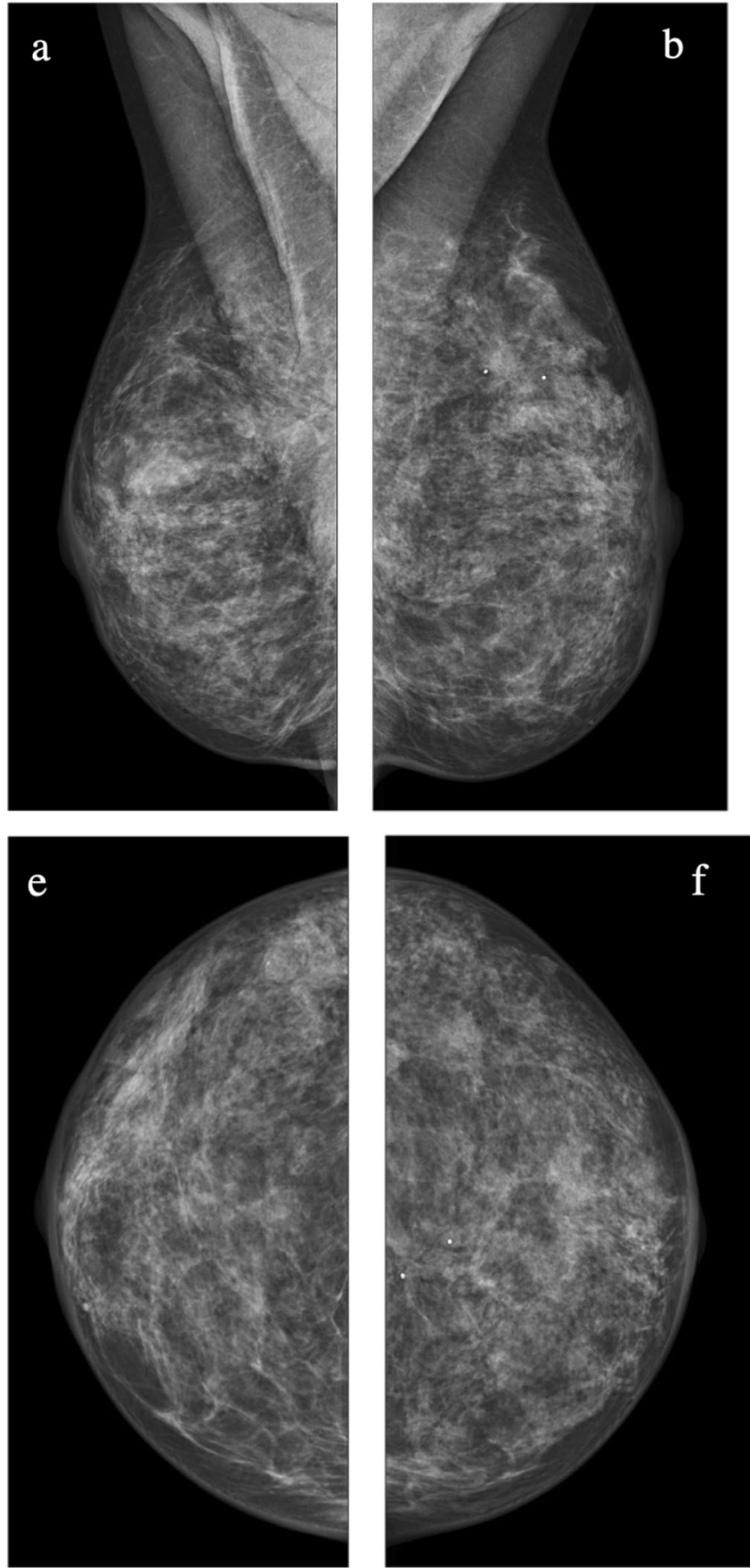

Fig. 2 A 51-year-old woman with extremely dense breasts. An architectural distortion was visible in the upper quadrant of the left breast (circle). The lesion was detected and classified as probably benign on digital mammography (DM) by one in four readers and suspicious by the other three readers. All four readers agreed in identifying the lesion as suspicious when evaluating one-view wide-angle digital breast tomosynthesis

differences in sensitivity between one-view WA-DBT with SM and two-view DM were not statistically significant ( $p=$ 0.226) (Fig. 1).

Overall specificity ranged from $60.2 \%$ for two-view DM to $69.9 \%$ for one-view DBT with SM. Specificity was better for
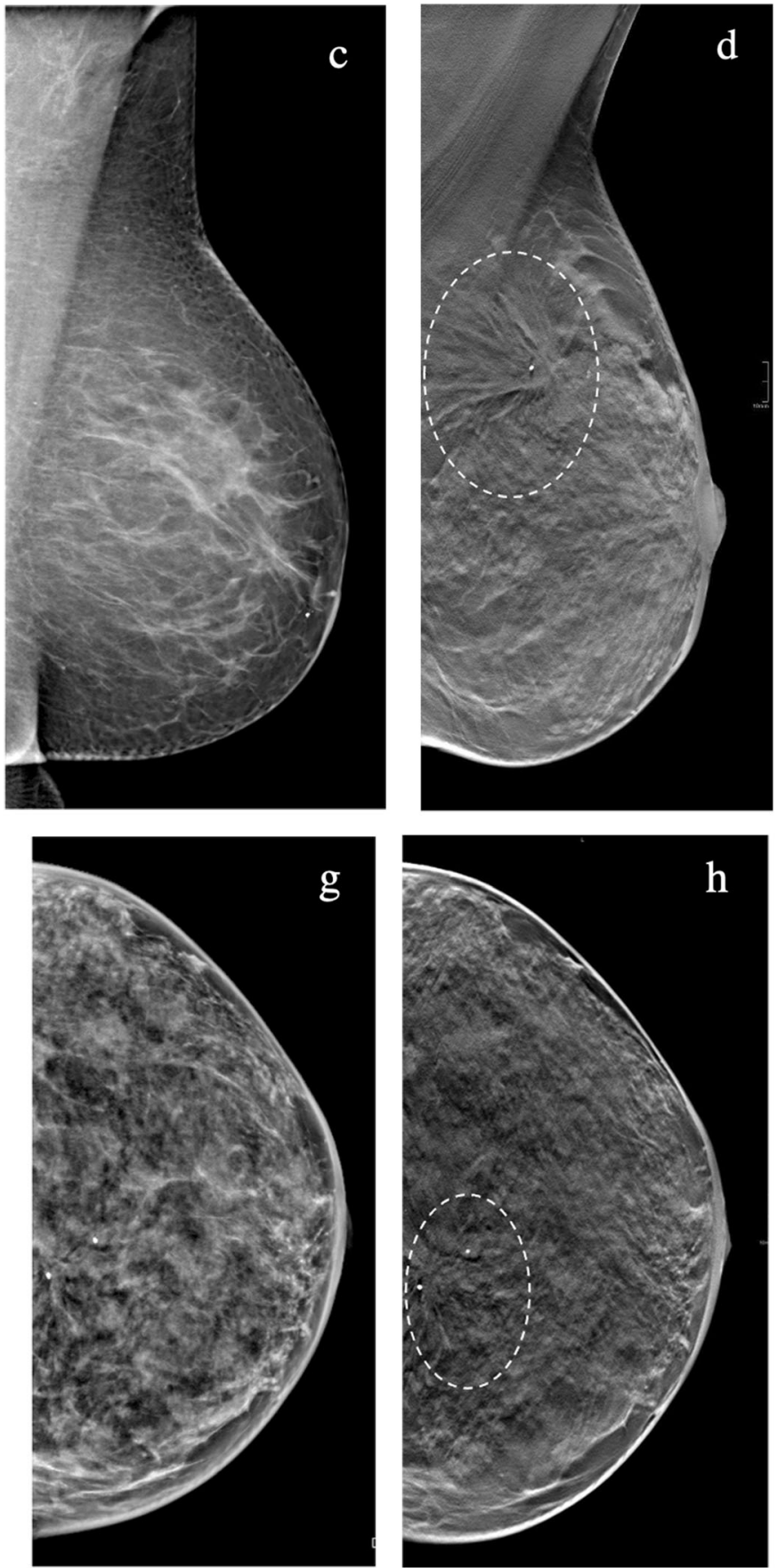

with synthetic mammography (WA-DBT with SM) and two-view WADBT with SM. The lesion was biopsied under ultrasound guidance and the diagnosis was fibrotic breast tissue with multiple papillomas. The absence of malignancy was confirmed at surgery. (a, b DM bilateral mediolateral oblique view (MLO); c SM MLO; d DBT MLO; e, f DM bilateral cranio-caudal view (CC); g SM CC; h DBT CC)

WA-DBT reading protocols, though the difference was not statistically significant compared to two-view DM (two-view DBT with SM, $p=0.104$; one-view DBT with SM, $p=0.061$ ) (Fig. 2).

The accuracy of two-view WA-DBT with SM and oneview WA-DBT did not differ significantly (overall $76.8 \%$ 
Table 5 Average accuracy of the three reading protocols for different breast densities, classified as according to the American College of Radiology (ACR) BI-RADS lexicon, and different lesion types

\begin{tabular}{llll}
\hline Accuracy & 2v-DM \% (95\% CI) & 1v-WA-DBT/SM \% (95\% CI) & 2v-WA-DBT/SM \% (95\% CI) \\
\hline ACR a & $83.3(71.1-91.1)$ & $83.5(70.0-91.7)$ & $88.8(76.2-95.1)$ \\
ACR b & $79.3(69.7-86.5)$ & $84.1(75.1-90.3)$ & $87.5(79.6-92.6)$ \\
ACR c & $72.8(64.7-79.6)$ & $84.4(77.1-89.6)$ & $81.5(74.9-86.7)$ \\
ACR d & $60.6(44.9-74.4)$ & $69.0(55.9-79.7)$ & $66.7(50.8-79.5)$ \\
Mass & $83.7(77.3-88.6)$ & $86.8(80.6-91.2)$ & $90.8(85.5-94.3)$ \\
Microcalcifications & $65.5(53.8-75.5)$ & $78.9(68.1-86.7)$ & $74.7(63.3-83.5)$ \\
Architectural distortion & $64.1(39.9-82.8)$ & $71.3(42.7-89.2)$ & $56.9(36.5-75.2)$ \\
Asymmetry & $40.7(13.8-74.6)$ & $61.0(25.1-88.0)$ & $72.5(43.7-89.9)$ \\
\hline
\end{tabular}

$2 v$-DM two-view digital mammography, $1 v$-WA-DBT/SM one-view wide-angle digital breast tomosynthesis with synthetic mammography, $2 v-W A-D B T /$ $S M$ two-view wide-angle digital breast tomosynthesis with synthetic mammography

and $75.8 \%$, respectively); however, both were significantly higher than two-view DM (67.5\%) (two-view WA-DBT with SM: $p<0.001$, one-view WA-DBT with SM: $p=0.003)$.

GEE showed that sensitivity and accuracy were dependent on the reading protocol used (both $p<0.001$ ), but not on the reader ( $p=0.162$ and $p=0.334$, respectively). In contrast, variations in specificity were not dependent on the reader $(p$ $=0.063)$ or the reading protocol $(p=0.059)$.

Inter-reader agreement in the BI-RADS assessment was fair to moderate with all three reading modalities: 0.413 for DM, 0.383 for two-view WA-DBT with SM, and 0.404 for one-view WA-DBT with SM.

\section{Effect of breast density and lesion type}

Accuracy decreased with increasing breast density for all three reading protocols and all four readers (Table 5). One-view and two-view WA-DBT with SM performed better than DM, regardless of breast density. Multivariate analysis showed that breast density significantly influenced the accuracy, regardless of the reading protocol $(p=0.002)$.

Accuracy varied for different lesion types, and multivariate analysis showed that the reading protocols and lesion type significantly affected accuracy $(p=0.024)$. Overall accuracy was higher with two-view WA-DBT with SM for masses and asymmetries, and it was higher with one-view WA-DBT with SM for calcifications and architectural distortions. DM did not outperform WA-DBT for any of the lesion types (Table 5).

\section{Lesion conspicuity, reading times, and radiation dose}

Average conspicuity assigned by the four readers was 9.26 for DM, 9.42 for one-view WA-DBT with SM, and 9.45 for twoview WA-DBT with SM. Lesion conspicuity for DM was significantly lower compared to both one-view and twoview WA-DBT with $\mathrm{SM}$ ( $p=0.011$ at multivariate analysis).
Average reading time for the four readers was $48.1 \mathrm{~s}$ for $\mathrm{DM}, 63.2 \mathrm{~s}$ for one-view WA-DBT with SM, and $75.1 \mathrm{~s}$ for two-view WA-DBT with SM. Reading times for DM were significantly lower compared to one-view and two-view WA-DBT with SM $(p<0.001)$. Reading times for one-view WA-DBT with SM were significantly lower than for twoview WA-DBT with SM $(p<0.001)$.

The median radiation dose of the DM views was $1.04 \mathrm{mGy}$ (95\% CI, 0.58-2.28 mGy). As the dose factor for the WADBT views was set to 2.0, this resulted in a similar dose per breast for the two-view DM and the one-view WA-DBT with SM (2.08 mGy; 95\% CI, 1.16-4.56 mGy). Two-view WADBT with SM had a twofold increased dose compared to the other two protocols (4.16 mGy; 95\% CI 2.32-9.12 mGy).

\section{Discussion}

The results of our study demonstrate that, in an assessment setting, two-view WA-DBT with SM achieves a higher detection rate and diagnostic performance compared to two-view DM. One-view WA-DBT with SM had a higher diagnostic accuracy compared to two-view DM, but there was no improvement in the detection rate. Furthermore, two-view WA-DBT with SM had a higher detection rate, but no significant improvement in diagnostic accuracy compared to oneview WA-DBT with SM.

The addition of two-view DBT to two-view DM allows for a significant increase in lesion detection rate [6, 21-24], but results in a relevant increase in radiation exposure $[8,9]$. The availability of SM eliminates the need to acquire DM, consequently reducing the radiation exposure, while maintaining a detection rate comparable to that of two-view DM with DBT [6, 19, 25]. To further reduce radiation exposure, some studies have suggested using WA-DBT only in the mediolateral oblique view. Zackrisson et al [13, 15] showed that one-view WA-DBT with or without the addition of one-view (cranio- 
caudal) DM can outperform two-view DM in the screening setting. Similar results were found by Rodriguez-Ruiz et al [14] in a cancer-enriched population. SM was not available for the analysis in any of these studies. The use of one-view WA-DBT with SM could reduce the radiation dose by more than $50 \%$ compared to two-view DBT with DM [9, 19].

In our analysis, we found a marginal improvement in accuracy when evaluating one-view WA-DBT with SM compared to two-view DM, and a more evident improvement when using two-view WA-DBT with SM. The detection rate, sensitivity, and accuracy improved when two-view WA-DBT and SM were available. Our results are in-line with a review that analyzed studies comparing one-view and two-view DBT with two-view DM, and underlined that the improvements were more substantial and conspicuous when two-view DBT was available [22]. In this analysis [22], one-view DBT with SM was not considered, but our results indicate that the same conclusions reached for one-view and two-view DBT with DM are also applicable to one-view and two-view DBT with SM.

Overall, it seems that two-view DBT is more relevant in an assessment setting, where it is crucial to upgrade or downgrade a previous finding and to detect multiple lesions. In a screening setting, one-view WA-DBT protocols seem to be sufficient to significantly increase the cancer detection rate and improve the screening performance, compared to DM alone $[13,15]$. In clinical practice, the evidence suggests that one-view WADBT (mediolateral oblique) should be preferred in screening, as it allows a reduction in reading times and radiation dose, and the second view (cranio-caudal) should be always performed in those patients recalled after screening or who present with symptoms, as it allows an additional improvement in detection and diagnostic performance [26-29].

Specificity did not differ significantly between the three reading protocols. This is also in agreement with previous European studies, which showed a limited effect of DBT on specificity and false-positive rates $[14,15,23]$. In addition, a recent meta-analysis underlined that, while the evidence suggests that DBT can increase the cancer detection rate in screening, no significant reduction in recall rate can be found when considering the overall data [25]. The use of SM rather than DM could also negatively influence specificity: previous analyses have indicated that the difficulties in the interpretation of findings in the synthetic images might lead to an increase in false positives [10,30].

WA-DBT improved the performance particularly for breasts with scattered fibroglandular tissue (ACR b) and heterogeneously dense breasts (ACR c), while the improvement for fatty breasts (ACR a) and extremely dense breasts (ACR d) was only marginal. This is in agreement with the results by Zackrisson et al [15] using one-view WA-DBT, and with other studies performed with two-view narrow-angle DBT [3, 31], which also found that DBT was particularly relevant in intermediate breast densities (ACR b and $\mathrm{c}$ ).
The availability of WA-DBT improved the accuracy for all lesion types, and the availability of two-view was particularly useful for the characterization of masses and asymmetries. This is in agreement with the literature and underlines that the quasi3D information available from DBT significantly improves the evaluation of soft-tissue lesions [26, 27]. DBT, with and without $\mathrm{SM}$, also seems to ensure a performance at least comparable to DM for calcifications [32-34], although, particularly for calcifications, studies have suggested that the use of SM might lead to a higher rate of false-positive findings [30, 35, 36].

Inter-reader agreement for BI-RADS was fair to moderate with all imaging modalities. This is in-line with previous published works that showed moderate inter-reader agreement [37, 38]. As opposed to Galati et al [39], we did not find an improvement in the inter-reader agreement when DBT was available.

Lesion conspicuity was rated as very good for all imaging modalities, but it was significantly higher when WA-DBT was available. Both Mariscotti et al [29] and Murakami et al [40] have already shown that lesion conspicuity is comparable between SM and DM. Of note, SM can improve lesion visibility in dense breasts and for calcified lesions [40].

We found, consistent with the published literature [16], a significant increase in reading times when WA-DBT was evaluated. A reduction in reading time and, consequently, in radiologists' fatigue is essential in a screening setting, where high volumes of examinations have to be evaluated in a short period of time [16-18]. The increase in reading times can be acceptable in an assessment setting, particularly when considering the advantages of improved lesion detection and characterization.

Our study has some limitations. First, it is a retrospective study, performed in a single assessment center. As the only exclusion criterion applied was the absence of a standard of reference (histology or 2 years' follow-up), we believe our dataset reflects the reality of an assessment center. No inference regarding the usefulness of one-view WA-DBT in a screening setting should be made based on our results. Only 19 patients presented with multiple lesions, and, of these, only six with multiple malignant lesions. Thus, it was not possible to perform a sub-analysis on the added value of WA-DBT for the evaluation of multifocal or multicentric lesions.

In conclusion, one-view and two-view WA-DBT with SM can achieve a higher diagnostic performance compared to two-view DM in an assessment setting. The detection rate and sensitivity were highest with two-view WA-DBT with SM. In the assessment of breast lesions, two-view WA-DBT with SM should become a standard and should not be replaced by one-view WA-DBT with SM or two-view DM.

Funding Open access funding provided by Medical University of Vienna. This study has received funding by Siemens Healthcare GmBH. 


\section{Declarations}

Guarantor The scientific guarantor of this publication is Thomas H. Helbich.

Conflict of interest P.C., P.A.B, T.H., and M.B. have received speaker honoraria from Siemens Healthcare.

Statistics and biometry One of the authors (M.W.) has significant statistical expertise.

Informed consent Written informed consent was waived by the Institutional Review Board due to the retrospective nature of the study.

Ethical approval Institutional Review Board approval was obtained.

Study subjects or cohorts overlap The study cohort has been previously reported in Clauser P., et al, Investigative Radiology 2019 54(2); 83-88. https://doi.org/10.1097/RLI.0000000000000513.

\section{Methodology}

- retrospective

- cross-sectional study

- performed at one institution

Open Access This article is licensed under a Creative Commons Attribution 4.0 International License, which permits use, sharing, adaptation, distribution and reproduction in any medium or format, as long as you give appropriate credit to the original author(s) and the source, provide a link to the Creative Commons licence, and indicate if changes were made. The images or other third party material in this article are included in the article's Creative Commons licence, unless indicated otherwise in a credit line to the material. If material is not included in the article's Creative Commons licence and your intended use is not permitted by statutory regulation or exceeds the permitted use, you will need to obtain permission directly from the copyright holder. To view a copy of this licence, visit http://creativecommons.org/licenses/by/4.0/.

\section{References}

1. Ciatto S, Houssami N, Bernardi D et al (2013) Integration of 3D digital mammography with tomosynthesis for population breastcancer screening (STORM): a prospective comparison study. Lancet Oncol 14:583-589. https://doi.org/10.1016/S14702045(13)70134-7

2. Friedewald SM, Rafferty EA, Rose SL et al (2014) Breast cancer screening using tomosynthesis in combination with digital mammography. JAMA 311:2499-2507. https://doi.org/10.1001/jama. 2014.6095

3. Gilbert FJ, Tucker L, Gillan MG et al (2015) The TOMMY trial: a comparison of TOMosynthesis with digital MammographY in the UK NHS Breast Screening Programme-a multicentre retrospective reading study comparing the diagnostic performance of digital breast tomosynthesis and digital mammography with digital mammography alone. Health Technol Assess 19:1-xxv, 1-136. https:// doi.org/10.3310/hta19040

4. Skaane P, Bandos AI, Niklason LT et al (2019) Digital mammography versus digital mammography plus tomosynthesis in breast cancer screening: the Oslo Tomosynthesis Screening Trial. Radiology 291:23-30. https://doi.org/10.1148/radiol.2019182394
5. Houssami N (2015) Digital breast tomosynthesis (3Dmammography) screening: data and implications for population screening. Expert Rev Med Devices 12:377-379. https://doi.org/ 10.1586/17434440.2015.1028362

6. Marinovich ML, Hunter KE, Macaskill P, Houssami N (2018) Breast cancer screening using tomosynthesis or mammography: a meta-analysis of cancer detection and recall. J Natl Cancer Inst 110: 942-949. https://doi.org/10.1093/jnci/djy121

7. Conant EF, Zuckerman SP, McDonald ES et al (2020) Five consecutive years of screening with digital breast tomosynthesis: outcomes by screening year and round. Radiology 295:285-293. https://doi.org/10.1148/radiol.2020191751

8. Svahn TM, Houssami N, Sechopoulos I, Mattsson S (2015) Review of radiation dose estimates in digital breast tomosynthesis relative to those in two-view full-field digital mammography. Breast 24:9399. https://doi.org/10.1016/j.breast.2014.12.002

9. Gennaro G, Bernardi D, Houssami N (2017) Radiation dose with digital breast tomosynthesis compared to digital mammography: per-view analysis. Eur Radiol. https://doi.org/10.1007/s00330017-5024-4

10. Ratanaprasatporn L, Chikarmane SA, Giess CS (2017) Strengths and weaknesses of synthetic mammography in screening. Radiographics 37:1913-1927. https://doi.org/10.1148/rg. 2017170032

11. Maldera A, De Marco P, Colombo PE et al (2017) Digital breast tomosynthesis: dose and image quality assessment. Phys Med 33: 56-67. https://doi.org/10.1016/j.ejmp.2016.12.004

12. Sechopoulos I (2013) A review of breast tomosynthesis. Part I. The image acquisition process. Med Phys 40:014301. https://doi.org/10. $1118 / 1.4770279$

13. Lång K, Andersson I, Rosso A et al (2016) Performance of oneview breast tomosynthesis as a stand-alone breast cancer screening modality: results from the Malmö Breast Tomosynthesis Screening Trial, a population-based study. Eur Radiol 26:184-190. https://doi. org/10.1007/s00330-015-3803-3

14. Rodriguez-Ruiz A, Gubern-Merida A, Imhof-Tas M et al (2017) One-view digital breast tomosynthesis as a stand-alone modality for breast cancer detection: do we need more? Eur Radiol. https://doi. org/10.1007/s00330-017-5167-3

15. Zackrisson S, Lång K, Rosso A et al (2018) One-view breast tomosynthesis versus two-view mammography in the Malmö Breast Tomosynthesis Screening Trial (MBTST): a prospective, population-based, diagnostic accuracy study. Lancet Oncol 19: 1493-1503. https://doi.org/10.1016/S1470-2045(18)30521-7

16. Bernardi D, Ciatto S, Pellegrini M et al (2012) Application of breast tomosynthesis in screening: incremental effect on mammography acquisition and reading time. Br J Radiol 85:e1174-e1178. https:// doi.org/10.1259/bjr/19385909

17. Dang PA, Freer PE, Humphrey KL et al (2014) Addition of tomosynthesis to conventional digital mammography: effect on image interpretation time of screening examinations. Radiology 270: 49-56. https://doi.org/10.1148/radiol.13130765

18. Iotti V, Giorgi Rossi P, Nitrosi A et al (2019) Comparing two visualization protocols for tomosynthesis in screening: specificity and sensitivity of slabs versus planes plus slabs. Eur Radiol 29: 3802-3811. https://doi.org/10.1007/s00330-018-5978-x

19. Clauser P, Baltzer PAT, Kapetas P et al (2019) Synthetic 2dimensional mammography can replace digital mammography as an adjunct to wide-angle digital breast tomosynthesis. Invest Radiol 54:83-88. https://doi.org/10.1097/RLI.0000000000000513

20. D’Orsi Carl J, Sickles EA, Mendelson EB, Morris EA (2013) ACR BI-RADS® Atlas, Breast Imaging Reporting and Data System, 5th edn. American College of Radiology, Reston

21. Wallis MG, Moa E, Zanca F et al (2012) Two-view and single-view tomosynthesis versus full-field digital mammography: high- 
resolution X-ray imaging observer study. Radiology 262:788-796. https://doi.org/10.1148/radiol.11103514

22. Svahn TM, Houssami N (2015) Digital breast tomosynthesis in one or two views as a replacement or adjunct technique to full-field digital mammography. Radiat Prot Dosimetry 165:314-320. https://doi.org/10.1093/rpd/ncv078

23. Gilbert FJ, Tucker L, Young KC (2016) Digital breast tomosynthesis (DBT): a review of the evidence for use as a screening tool. Clin Radiol 71:141-150. https://doi.org/10.1016/j.crad. 2015.11.008

24. Sprague BL, Coley RY, Kerlikowske K et al (2020) Assessment of radiologist performance in breast cancer screening using digital breast tomosynthesis vs digital mammography. JAMA Netw Open 3:e201759. https://doi.org/10.1001/jamanetworkopen. 2020.1759

25. Giampietro RR, Cabral MVG, Lima SAM et al (2020) Accuracy and effectiveness of mammography versus mammography and tomosynthesis for population-based breast cancer screening: a systematic review and meta-analysis. Sci Rep 10:7991. https://doi.org/ 10.1038/s41598-020-64802-x

26. Bahl M, Mercaldo S, Vijapura CA et al (2019) Comparison of performance metrics with digital 2D versus tomosynthesis mammography in the diagnostic setting. Eur Radiol 29:477-484. https:// doi.org/10.1007/s00330-018-5596-7

27. Zuley ML, Bandos AI, Ganott MA et al (2013) Digital breast tomosynthesis versus supplemental diagnostic mammographic views for evaluation of noncalcified breast lesions. Radiology 266:89-95. https://doi.org/10.1148/radiol.12120552

28. Fontaine M, Tourasse C, Pages E et al (2019) Local tumor staging of breast cancer: digital mammography versus digital mammography plus tomosynthesis. Radiology 291:594-603. https://doi.org/ 10.1148/radiol.2019182457

29. Mariscotti G, Durando M, Houssami N et al (2017) Comparison of synthetic mammography, reconstructed from digital breast tomosynthesis, and digital mammography: evaluation of lesion conspicuity and BI-RADS assessment categories. Breast Cancer Res Treat 166:765-773. https://doi.org/10.1007/s10549-0174458-3

30. Caumo F, Zorzi M, Brunelli S et al (2017) Digital breast tomosynthesis with synthesized two-dimensional images versus full-field digital mammography for population screening: outcomes from the Verona Screening Program. Radiology 170745. https://doi.org/10.1148/radiol.2017170745

31. Østerås BH, Martinsen ACT, Gullien R, Skaane P (2019) Digital mammography versus breast tomosynthesis: impact of breast density on diagnostic performance in population-based screening. Radiology 293:60-68. https://doi.org/10.1148/radiol.2019190425
32. Choi JS, Han B-K, Ko EY et al (2019) Comparison of synthetic and digital mammography with digital breast tomosynthesis or alone for the detection and classification of microcalcifications. Eur Radiol 29:319-329. https://doi.org/10.1007/s00330-018-5585-x

33. Clauser P, Nagl G, Helbich TH et al (2016) Diagnostic performance of digital breast tomosynthesis with a wide scan angle compared to full-field digital mammography for the detection and characterization of microcalcifications. Eur J Radiol 85:2161-2168. https://doi. org/10.1016/j.ejrad.2016.10.004

34. Lai Y-C, Ray KM, Lee AY et al (2018) Microcalcifications detected at screening mammography: synthetic mammography and digital breast tomosynthesis versus digital mammography. Radiology 289:630-638. https://doi.org/10.1148/radiol.2018181180

35. Zhu H, Polat D, Evans $P$ et al (2020) Is there a difference in the diagnostic outcomes of calcifications initially identified on synthetic tomosynthesis versus full-field digital mammography screening? Eur J Radiol 133:109365. https://doi.org/10.1016/j.ejrad.2020. 109365

36. Dodelzon K, Simon K, Dou E et al (2020) Performance of 2D synthetic mammography versus digital mammography in the detection of microcalcifications at screening. AJR Am J Roentgenol 214: 1436-1444. https://doi.org/10.2214/AJR.19.21598

37. Berg WA, Campassi C, Langenberg P, Sexton MJ (2000) Breast Imaging Reporting and Data System: inter- and intraobserver variability in feature analysis and final assessment. AJR Am J Roentgenol 174:1769-1777. https://doi.org/10.2214/ajr.174.6. 1741769

38. Lee AY, Wisner DJ, Aminololama-Shakeri S et al (2017) Interreader variability in the use of BI-RADS descriptors for suspicious findings on diagnostic mammography: a multi-institution study of 10 academic radiologists. Acad Radiol 24:60-66. https://doi.org/ 10.1016/j.acra.2016.09.010

39. Galati F, Marzocca F, Bassetti E et al (2017) Added value of digital breast tomosynthesis combined with digital mammography according to reader agreement: changes in BI-RADS rate and follow-up management. Breast Care (Basel) 12:218-222. https://doi.org/10. $1159 / 000477537$

40. Murakami R, Uchiyama N, Tani H et al (2020) Comparative analysis between synthetic mammography reconstructed from digital breast tomosynthesis and full-field digital mammography for breast cancer detection and visibility. Eur J Radiol Open 7:100207. https:// doi.org/10.1016/j.ejro.2019.12.001

Publisher's note Springer Nature remains neutral with regard to jurisdictional claims in published maps and institutional affiliations. 